\title{
Dynamical Evolution of Globular Cluster Systems in Clusters of Galaxies: The Case of NGC 1404 in the Fornax Cluster
}

\author{
Kenji Bekki, Warrick J. Couch \\ School of Physics, University of New South Wales, Sydney, NSW, 2052, \\ Australia
}

\author{
Duncan A. Forbes and M. A. Beasley \\ Centre for Astrophysics \& Supercomputing, Swinburne University of \\ Technology, Hawthorn, VIC, 3122, Australia
}

\begin{abstract}
We investigate, via numerical simulations, the tidal stripping and accretion of globular clusters (GCs). In particular, we focus on creating models that simulate the situation for the GC systems of NGC 1404 and NGC 1399 in the Fornax cluster, which have poor (specific frequency $\left.S_{N} \sim 2\right)$ and rich $\left(S_{N} \sim\right.$ 10) GC systems respectively. We initially assign NGC 1404 in our simulation a typical $S_{\mathrm{N}}(\sim 5)$ for cluster ellipticals, and find that its GC system can only be reduced through stripping to the presently observed value, if its orbit is highly eccentric (with orbital eccentricity of $>0.5$ ) and if the initial scale length of the GCs system is about twice as large as the effective radius of NGC 1404 itself. These stripped GCs can be said to have formed a 'tidal stream' of intra-cluster globular clusters (ICGCs) orbiting the center of Fornax cluster (many of which would be assigned to NGC 1399 in an imaging study). The physical properties of these GCs (e.g., number, radial distribution, and kinematics) depend on the orbit and initial distribution of GCs in NGC 1404. Our simulations also predict a trend for $S_{\mathrm{N}}$ to rise with increasing cluster-centric distance - a trend for which there is some observational support in the Fornax cluster. We demonstrate that since the kinematical properties of ICGCs formed by tidal stripping in the cluster tidal field depend strongly on the orbits of their previous host galaxies, observations of ICGC kinematics provides a new method for probing galaxy dynamics in a cluster.
\end{abstract}

\section{Observable evidence for the tidal stripping scenario for low $S_{\mathrm{N}}$ cluster ellipticals}

We suggest that the following three are the observable evidences: First is the ratio of $S_{\mathrm{N}}$ within $1-2 R_{\mathrm{e}}$ to that within $5-10 R_{\mathrm{e}}$. The second is the formation of an elongated or flattened distribution (or "tidal stream") of ICGCs along the orbit of their previous host galaxy. The third is the statistical correlation between the distance of an elliptical galaxy from the center of a cluster and the $S_{\mathrm{N}}$ of the galaxy (more details are given by Bekki et al. 2003 MNRAS, 344, 1334). 